\title{
B-lymphocyte Crossmatch Measurement
}

National Cancer Institute

\section{Source}

National Cancer Institute. B-Iymphocyte Crossmatch Measurement. NCI Thesaurus. Code C128951.

The determination of the HLA histocompatibility between the donor and the study subject by examining the presence or absence of the subject's anti-HLA antibody reactivity towards HLA antigens expressed on the donor B-lymphocytes. 